\title{
SIRT4 functions as a tumor suppressor in prostate cancer by inducing apoptosis and inhibiting glutamine metabolism
}

\section{Liangliang Cai}

Guangxi University of Traditional Chinese Medicine

\section{Zhuhui Ge}

First Hospital of Ninghai County

\section{Yunqiu Xu}

Department of Neurosurgery, the First Affiliated Hospital, Wenzhou Medical University, Wenzhou, China.

\section{Pingliang Sun}

Guangxi University of Traditional Chinese Medicine

Guoyu Huang ( $\sim$ huangguoyu.greg@gmail.com )

Guangxi University of Traditional Chinese Medicine

\section{Article}

Keywords: SIRT4, prostate cancer, Gleason Score, proliferation, glutamine

Posted Date: May 27th, 2022

DOI: https://doi.org/10.21203/rs.3.rs-1417679/v2

License: (9) This work is licensed under a Creative Commons Attribution 4.0 International License. Read Full License 


\section{Abstract}

SIRT4, localized in the mitochondria, is an nicotinamide adenine dinucleotide (NAD+) -dependent adenosine diphosphate (ADP) -ribosyltransferase and is one of the least characterized members of the sirtuin family. Although it is well known that it shows deacetylase activity for energy metabolism, little is understood about its function in tumorigenesis. Recent research suggests that SIRT4 may work as both a tumor suppressor gene and an oncogene. However, the clinical significance of SIRT4 in prostate cancer remains unknown. In this study, we evaluated SIRT4 protein levels in cancerous prostate tissue and corresponding non-tumor prostate tissue via immunohistochemical staining on a tissue microarray including tissues from 89 prostate cancer patients. The association between SIRT4 expression and Gleason score was also determined. Further, shSIRT4 or stable prostate cancer cell lines (22RV1) overexpressing SIRT4 were constructed via lentiviral infection. Using Cell-Counting Kit-8 (CCK-8) assay, wound healing assay, migration, and invasion and apoptosis assays, the effects of SIRT4 on the migration, invasion ability, and proliferation of prostate cancer cells were investigated. We also determined the effect of SIRT4 on glutamine metabolism in 22RV1 cells. We found the protein levels of SIRT4 in prostate cancer tissues were significantly lower than those in their non-neoplastic tissue counterparts $(P<0.01)$; a lower SIRT4 level was also significantly associated with a higher Gleason score $(P<0.01)$. SIRT4 suppressed the migration, invasion capabilities, and proliferation of prostate cancer cells and induced cellular apoptosis. Furthermore, the invasion and migration of 22RV1 cells were mechanistically inhibited by SIRT4 via glutamine metabolism inhibition. In conclusion, the present study's findings showed that SIRT4 protein levels are significantly associated with the Gleason score in patients with prostate cancer, and SIRT4 exerts a tumor-suppressive effect on prostate cancer cells by inhibiting glutamine metabolism. Thus, SIRT4 may serve as a potential novel therapeutic target for prostate cancer.

\section{Introduction}

Prostate cancer is the most common type of cancer diagnosed in the male population and the second leading cause of cancer-related deaths among males in the United States and other western countries [1]. According to the American Cancer Society, approximately 160,000 new cases of prostate cancer were reported in the United States in 2017, of which 32,050 patients died of the disease [2]. Prostate cancer develops through a series of defined steps, including invasive carcinoma, prostate intraepithelial neoplasia (PIN), and hormone-dependent or -independent metastasis. Although different stages of prostate cancer have histologically been well defined, relatively few underlying molecular mechanisms contributing to the initiation and development of prostate cancer are known.

The sirtuin (SIRT) protein family is a group of $\mathrm{NAD}^{+}$dependent deacetylases and ADP ribosyltransferases. Humans encode seven sirtuin isoforms (SIRT1-SIRT7) and these have been recognized to play essential roles in stress resistance, energy homeostasis, and aging [3]. SIRT4, an $N A D^{+}$-dependent ADP-ribosyltransferases localized in the mitochondria, interacts with and represses glutamate dehydrogenase (GDH) activity via mono-ADP-ribosylation [4], and facilitates glucose homeostasis, insulin secretion, and fatty acid oxidation [4-7]. Recent studies indicate that mitochondrial 
SIRT4 exhibits tumor-suppressing activities by promoting genomic stability [7-11]. Studies on glutamine metabolism regulation have also shown that SIRT4 may also function as an oncogene $[12,13]$. However, there few studies about the effects of SIRT4 on prostate cancer cells. Thus, SIRT4's role in prostate cancer stays elusive.

In this study, we evaluated the associations between immunohistochemical SIRT4 protein expression in prostate cancer tissue and Gleason score to determine the clinical importance of SIRT4 expression in patients with prostate cancer. Moreover, we investigated the in vitro effects of SIRT4 on prostate cancer cell proliferation, migration, and invasion, as well as prostate cancer cell apoptosis, by inhibiting and overexpressing SIRT4 gene in 22RV1 cells. Finally, we investigated the effect of SIRT4 on L-Glutamine metabolism and its role in inhibiting the function of prostate cancer cells.

\section{Materials And Methods}

Ethical approval was approved by the local Ethics Committee of the first affiliated hospital of Wenzhou Medical University (Wenzhou, China) in view of the retrospective nature of the study and was conducted in accordance with the principles of the Declaration of Helsinki.

\section{Patients and tissue samples}

Tumor tissue specimens from 89 patients with prostate cancer who underwent surgery were acquired from the First Affiliated Hospital of Wenzhou Medical University between February 2011 and March 2016. The mean age of the patients was 70 years (range, 55-90 years). Informed consent was obtained from all participants. No patient underwent preoperative radiotherapy or chemotherapy before surgery. All patients were diagnosed with pathological type prostate adenocarcinoma. The patients' Gleason scores were obtained from the American Joint Committee on Cancer (AJCC, 7th edition) data.

Tissue microarray (TMA) chips were obtained commercially (Superchip Inc., Shanghai, China). The TMA contained 89 prostate cancer patient specimens including tumor tissue samples and their corresponding adjacent non-neoplastic tissue specimens. A tissue cylinder (diameter, $2 \mathrm{~mm}$ ) was stamped out from morphologically representative areas of each donor block and transferred to a recipient paraffin block. Tissue microarray blocks were $2.0 \mathrm{~mm}$ in diameter, and all points were covered with paraffin wax.

\section{Immunohistochemical analysis}

The immunohistochemical assay was performed on TMA chips. The chip was deparaffinized in xylene twice for 5 min at room temperature (RT) and then rehydrated in successively graded concentrations of ethanol at $100 \%, 95 \%, 85 \%$, and $70 \%$, respectively for $5 \mathrm{~min}$. Antigen retrieval was performed by heating the sections at $100^{\circ} \mathrm{C}$ for $30 \mathrm{~min}$ in citrate buffer $(0.05 \%$ Tween 20 and $10 \mathrm{mM}$ citrate, pH 6.0) at $170 \mathrm{kPa}$ at $120^{\circ} \mathrm{C}$ for $5 \mathrm{~min}$. Subsequently, endogenous peroxidase activity was blocked by incubation in $0.3 \%$ $\mathrm{H}_{2} \mathrm{O}_{2}$ in Tris- $\mathrm{HCl}$ buffer for 15 min at RT. The chip was then washed 3 times and incubated with a polyclonal rabbit anti-SIRT 4 antibody (HPA029692, 1:400, Sigma, USA) at $4^{\circ} \mathrm{C}$ in a refrigerator for more 
than $8 \mathrm{~h}$. Subsequently, a secondary antibody was added to the chip using the GTVision Kit (Gene Tech Inc., Shanghai, China) and the chip was incubated, as per the manufacturer's instructions. The microarray chip section was then consecutively stained with diaminobenzidine (DAB) and hematoxylin. Next, the chip was dehydrated and sealed with a coverslip. Tissues treated with only a diluent (without antibody) were used as negative controls.

Tissue wafer scanning and analysis method:

Tissue chip scanner model Pannoramic MIDI, manufacturer: 3D HISTECH (Budapest, Hungary): The tissue chip section gradually moves under the scanner lens. The moving edge is imaged, and the information on the tissue section is scanned and imaged to create a file. The file contains all the tissue information available on the tissue section. The file can be enlarged 1-400 times in the Pannoramic viewer software. Further, any part of the tissue section can be imaged. The Quant center is an analysis software program associated with the Pannoramic viewer. On scan completion, the TMA software of the Quant center analysis software can be run after setting the diameter of the wafer tissue and the number of rows and columns. The software then generates a number. The nuclei in the tissue sections that were strongly positive were colored dark brown, those moderately positive were brown yellow, those weakly positive were pale yellow, and those negative were blue using the densito quant software in Quant center. The area (unit: pixel), the percentage of positive staining, and final last histochemical score (H-SCORE) were analyzed for each tissue site. The formula for calculating the H-SCORE was as follows: $\mathrm{H}$-SCORE = $\sum(\mathrm{Pi} \times \mathrm{I})=($ percentage of weakly positive cells $\times 1)($ percentage of moderately positive cells $\times 2)$ (percentage of strongly positive cells $\times 3$ ), with pi representing the percentage of the positive cells in the tissue section and $i$ representing the intensity of the staining. The H-SCORE score ranged from 0 to 300 , with a higher score indicating a stronger positive score [14, 15].

\section{Cell lines and culture conditions}

A human prostate cancer cell line, 22RV1, was procured from Shanghai Institute of Cell Biology, Chinese Academy of Sciences in June 2020. The cell bank use four pairs of primers DXS52, Apo-B, MD17S5 and D2S44 to monitor the variation of cell lines during passage. The cells were last tested in June 2020, and the cell experiment ends within half a year after the cell is purchased. Cells were maintained in Dulbecco's modified Eagle's medium (DMEM; Gibco, USA) supplemented with $10 \%$ fetal bovine serum (Gibco, USA) and penicillin/streptomycin (Gibco, USA), and incubated at $37^{\circ} \mathrm{C}$ with $5 \% \mathrm{CO}_{2}$.

\section{Vector and virus production}

We design three siRNA sequences targeted to SIRT4, they were 5'-GCGTGTCTGAAACTGAATTCT', 5'GCTCCTGATGGTGACGTCTTTCTCT-3' and 5'-GCGTTCAATGTGGAGGCCATCTGAA-3', respectively. The negative control sequence was 5'-TGTCACTCTCCGGAACGTT-3'. We purchased the lentivirus vector pHBLV-CMVIE-ZsGreen-T2A-Puro overexpressing SIRT4 and shSIRT4 from biotechnology company (Hanbio, Shanghai, China). The final titer of the lentivirus and negative control virus was $2 \times 10^{8} \mathrm{PFU} / \mathrm{ml}$. Stable overexpression of SIRT4 or shSIRT4 was achieved by transfecting 22RV1 cells with lentivirus for 
$72 \mathrm{~h}$; colonies were isolated using puromycin for two weeks. Lipofectamine2000 (Thermo Fisher Science) was used as the transfection reagent.

\section{Reverse transcription (RT)-PCR}

Total RNA from the tissues or cells was extracted using the TRIzol reagent (Invitrogen, USA). Further, 500 ng of cDNA was synthesized using a reverse transcription kit (PrimeScriptTM RT Master Mix, TaKaRa, Japan). Then, qRT-PCR was performed with thrice-diluted cDNA using the qRT-PCR Kit (SYBR® Premix Ex $\mathrm{Taq}^{\mathrm{TM}}$ aqR KTaKaRa, Japan) on the DNA Engine Opticon 2 real-time detection system (BioRad). GAPDH was chosen as the internal reference gene. Primers for each gene were as follows: SIRT4 forward primer 5'- GCGAGAAACTTCGTAGGCTG - 3', reverse primer 5'- TCAGGACTTGGAAACGCTCT - 3'; GAPDH forward primer 5'-TCAAGAAGGTGGTGAAGCAGG - 3', reverse primer 5'- TCAAAGGTGGAGGAGTGGGT - 3'. The PCR reaction conditions were as follows: 2 min at $94^{\circ} \mathrm{C}$; followed by 40 cycles of $30 \mathrm{~s}$ at $94^{\circ} \mathrm{C}, 30 \mathrm{~s}$ at $57^{\circ} \mathrm{C}$, and $1 \mathrm{~min}$ at $72^{\circ} \mathrm{C}$; and ending with $5 \mathrm{~min}$ at $72^{\circ} \mathrm{C}$ and cooling at $4^{\circ} \mathrm{C}$. After the reaction was completed, the homogeneity of the PCR product was confirmed by analyzing the dissolution curve followed by analysis of relative gene expression by $2^{-\Delta \Delta} \mathrm{CT}$.

\section{Western blotting}

Cells were lysed with Ripa lysis buffer (Beyotime, China) supplemented with protease inhibitor cocktail (Beyotime, China). Cell lysates were separated by sodium dodecyl sulfate-polyacrylamide gel electrophoresis (SDS-PAGE) and immunoblotting. The primary antibodies used were rabbit anti-human SIRT4 polyclonal antibody (clone HPA029691, Sigma, USA), rabbit anti-human caspase3 (35/18 KDa) polyclonal antibody (9662, CST, USA), rabbit anti-human caspase9 (46 KDa) polyclonal antibody (103801-AP, Proteintech, China), rabbit anti-human p65 (65 KDa) monoclonal antibody (8242, Cell signaling, USA), rabbit anti-human p-p65 (65 KDa) monoclonal antibody (3033, Cell signaling, USA),rabbit antihuman matrix metalloproteinase 9 (MMP9) (78 KDa) polyclonal antibody (10375-2-AP, Proteintech, China), rabbit anti-human n-cadherin ( $99 \mathrm{KDa}$ ) monoclonal antibody (14472, CST, USA), rabbit antihuman e-cadherin (135 KDa) monoclonal antibody (Ab124397, Abcam, England), goat anti-rabbit detection antibody (ab97200, Abcam, England), rabbit anti-human Lamin B1 (67 KDa) polyclonal antibody (BA1228, Boster Biological Technology, China), and rabbit anti-human glyceraldehyde 3phosphate dehydrogenase (GAPDH) polyclonal antibody (AB-P-R 001, Goodhere, China).

\section{Biochemical assay}

For the analysis of GDH activity, the biochemical activity of these cell lines was analyzed using the Human Glutamate dehydrogenase (GLDH) test kit (A123, Jiancheng Bioengineering Institute, Nanjing, China).

\section{Cell proliferation activity}

Cells were inoculated in $96-$ well plates with a density of 1000 cells/well. Subsequently, $10 \mu \mathrm{L}$ of cell counting kit-8 (CCK-8) reagent (Dojindo, Japan) was added in each well followed by culturing in a $\mathrm{CO}_{2}$ incubator for $2 \mathrm{~h}$ and absorbance determination. The final concentration of the bis-2-(5-phenylacetamide- 
1,2,4-thiadiazol-2-yl)ethyl sulfide (BPTES; SML0601, Sigma, USA) was $10 \mu \mathrm{mol} / \mathrm{L}$. The final concentration of dimethyl a-ketoglutarate (DM-aKG; 349631, Sigma, USA) was $8 \mathrm{mmol} / \mathrm{L}$.

\section{Wound healing assay}

Wound healing assay was carried out to determine cell migration. Briefly, the SIRT4 overexpression, shSIRT4, and the corresponding negative virus group cells were seeded in a 6-well plate with a density of $5 \times 10^{5}$ per well and cultured for $10 \mathrm{~h}$. Following this, a 200-microlite tip perpendicular to the bottom of the plate was used to make a scratch line. Subsequently, the detached cells were washed with PBS and cultured in a serum-free medium. DM-aKG at a concentration of $4 \mathrm{mM}$ or $8 \mathrm{mM}$ was added after scratching in the DM-aKG group. In the experimental group, cell images in the scratched area after 0 and $24 \mathrm{~h}$ of culture, obtained using an optical microscope, were used to calculate the distance migrated by the cells.

\section{Cell Migration and invasion test}

In the migration test, cells were suspended in a serum-free medium with a density of $3 \times 10^{5} \mathrm{ml}$, and a 0.2-ml suspension was inoculated into the upper chamber of the Transwell plate (Corning Inc., Conning, New York, USA). The cells were filled with $0.6 \mathrm{ml}$ culture medium containing $10 \% \mathrm{FBS}$. The cells were then incubated at $37^{\circ} \mathrm{C}$ for $18 \mathrm{~h}$. The cells in the upper compartment were then wiped with a sterile cotton swab and the membrane was soaked in $4 \%$ paraformaldehyde to immobilize the cells in the lower compartment, followed by staining with $0.1 \%$ crystal violet. The cells were counted under an optical microscope (Olympus). In the invasion test, the superior chamber was precoated with BD Matrigel ${ }^{T M}$ Basement Membrane Matrigel (BD biosciences) before cell inoculation. The rest of the procedure was the same as that followed for the migration test. For DM- $₫ K G$ group, the culture medium with a $4 \mathrm{mM}$ or 8 mM DM- $₫ K G$ was adjusted after seeding.

\section{Flow cytometric analysis for apoptosis rate and cell cycle}

Cells were harvested by trypsinization, pelleted by centrifugation, and resuspended in PBS containing $3 \%$ fetal bovine serum. The cell apoptosis was performed with flow cytometry (C6, BD, USA) using annexin Vallophycocyanin (annexin V-APC) and 7-aminoactinomycin D (7-AAD) (BD, USA) staining. Apoptosis rate was only calculated at early apoptosis. The survival rate was calculated by cells not stained with annexin V-APC or 7-AAD. The apoptotic cells were analyzed by flow cytometry analysis using the Accuri C6 software program (BD, USA). Cell cycle was measured with Propidium lodide (PI)/RNase kit (BD, USA). The results were examined with the ModFit analysis software program (Verity Software House, Topsham, ME, USA).

\section{Cell immunofluorescence}

Immunofluorescence analysis were performed as described previously [16], using rabbit anti-human NFKB p65 polyclonal antibody (10745-1-AP, 1:50 dilution, Proteintech Group, China), rabbit anti-human Phospho-N F-KB p65 polyclonal antibody (3033S, 1:50 dilution, CST, USA), and an Cy3-conjugated goat 
anti-rabbit IgG secondary antibody (BA1032, 1:100 dilution, Boster Biological Technology, China). DAPI was used for staining of nuclei and to assess gross cell morphology.

\section{Statistical analysis}

The SPSS software package version 22.0 (SPSS, Inc., IBM, USA) was used for statistical analysis. All in vitro experiments were performed in triplicates. Data from three or more independent experiments are presented as the mean \pm standard deviation. The final scores of tumor tissue and non-tumor tissue were analyzed using a paired student's $t$ tests. Experiments unless specified were analyzed using the nonpaired $t$-test. $P<0.05$ (two-tailed) was considered statistically significant.

\section{Results}

\section{SIRT4 immunostaining intensity was significantly decreased in prostate cancer tissue}

On immunohistochemical staining of human prostate cancer and adjacent non-neoplastic tissues, we found that SIRT4 was predominantly localized in the cytoplasm. The immunostaining intensity of SIRT4 in the tumor tissue was notably lower (Fig. 1) than that in the adjacent non-neoplastic prostate tissues. The difference was statistically significant (Fig. 2A).

\section{The association of SIRT4 immunostaining intensity with Gleason score in prostate cancer}

To determine the clinical significance of SIRT4 expression in patients with prostate cancer, the relationship between SIRT4 expression and the Gleason score in prostate cancer was analyzed. A significant negative correlation was observed between SIRT4 expression and the Gleason score in prostate cancer in the present study (Fig. 2B). We found that a higher Gleason score was exhibited in patients with low expressions of SIRT4.

Effect of SIRT4 on the proliferation, migration, and invasion capabilities of the prostate cancer cell line

Through the pre-experiment, we found that the interference effect of siRNA sequence 5'GCGTTCAATGTGGAGGCCATCTGAA-3' is the best (data not show). We constructed stable strains with SIRT4 overexpression and suppression of 22RV1 prostate cancer cells via lentiviral infection and confirmed the results by RT-PCR (Fig. 3A) and Western Blotting (Fig. 3B). Cell proliferation assay revealed that SIRT4 overexpression significantly suppressed the proliferation of 22RV1 cells (Fig. 3C); in contrast, after SIRT4 inhibition, the proliferative activity of 22RV1 cells was significantly higher than that of the untransfected cells and negative controls (Fig. 3D). Moreover, cell migration assay showed that SIRT4 overexpression significantly lowered the wound-healing rate (Fig. 3E); the wound-healing rate, however, was accelerated on SIRT4 knockdown (Fig. 3F). Next, we found that invasion and migration abilities of 22RV1 cells were significantly reduced with SIRT4 overexpression (Fig. 3G and 3I), and significantly 
increased after SIRT4 inhibition (3H and Fig. 3J). Taken together, these results suggested that SIRT4 plays a critical role in determining the proliferation, migration, and invasion abilities of prostate cancer cells in vitro experiments.

\section{Overexpression of SIRT4 induces prostate cancer cell apoptosis}

Next, we explored the effects of SIRT4 on the proliferation of prostate cancer cells by inhibiting cell apoptosis. We found that apoptosis was significantly increased in 22RV1 cells with SIRT4 overexpression compared with that in the controls (Fig. 4A). To further investigate the molecular mechanism underlying SIRT4-induced apoptosis in 22RV1 cells, the expression of apoptotic regulatory proteins was detected by Western blotting following SIRT4 overexpression. SIRT4 overexpression resulted in a significant induction of caspase3 18 KDA and caspase9 expressions (Fig. 4B), which agreed with flow cytometry findings. We also found that SIRT4 overexpression reduced p65 levels in the cytoplasm (Fig. 4C) and p-p65 levels in the nucleus (Fig. 4D). A similar decline in p65 and p-p65 levels was noticed in 22RV1 cells with SIRT4 overexpression via immunofluorescence testing (Fig. 4E and 4F).

SIRT4 regulates the proliferation, migration, and invasion capabilities of prostate cancer cells via inhibition of glutamine metabolism

To further elucidate the possible mechanisms underlying SIRT4-induced inhibition of invasion and migration, the levels of epithelial-mesenchymal transition (EMT)-related proteins were evaluated by Western blotting. SIRT4 overexpression notably increased E-cadherin expression significantly but reduced MMP9 and N-cadherin expressions (Fig. 5A). These results suggest that SIRT4 plays a crucial role in the invasion and migration of prostate cancer cells.

Accumulating evidence has shown that SIRT4 overexpression suppresses GDH enzymatic activity and limits the metabolism of glutamine and glutamate, thus generating adenosine triphosphates (ATPs) [4]. We found that GDH activity was significantly suppressed in 22RV1 cells after SIRT4 overexpression (Fig. 5B), whereas GDH activity was markedly increased after SIRT4 knockdown (Fig. 5C). Glutamine is converted into glutamic acid by glutaminase activity, and later, glutamate is translated to a-Ketoglutaric acid (a-KG) through GDH or a transaminase coupling reaction [17]. a-KG is a crucial metabolic product of glutamine metabolism. For determining whether SIRT4 inhibits the invasion and migration of prostate carcinoma cells by inhibiting glutamine metabolism, we treated 22RV1 cells with DM-aKG and found that the reduced proliferation after SIRT4 overexpression was revoked (Fig. 5D). Further, we observed that when DM-aKG was added to the medium, a significant increasing was observed in the rate of wound healing in 22RV1 cells overexpressing SIRT4 (Fig. 5E); however, no change was observed in the wound healing rate in the negative control group on addition of DM-aKG (Fig. 5F). Similarly, the invasion rate of

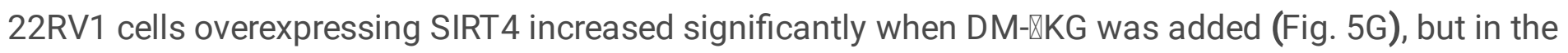
negative control cells, no significant change was observed (Fig. 5H). These results indicate that SIRT4 suppresses the proliferation, migration, and invasion capabilities of 22RV1 cells by inhibiting glutamine metabolism. 


\section{Discussion}

SIRT4 has been found to exhibit tumor suppressive role by virtue of its metabolic regulatory activity. However, there is still only limited information regarding the clinical significance and function of SIRT4 in tumorigenesis, and all agree studies suggest that it plays a role in inhibiting cancer genes. Recently, Jeong demonstrated that SIRT4 inhibits the formation of tumors by inhibiting glutamine metabolism [7]. Further, SIRT4 overexpression inhibits the proliferation ability of HeLa cells, and SIRT4 knockdown in MEF cells inhibits large tumor development in nude mice in vivo. Nevertheless, SIRT4-knockout mice spontaneously developed cancers of liver, lung, and breast. Furthermore, Csibi et al. showed that SIRT4 overexpression represses the proliferation ability of DU145 and DLD-1 cell lines [8]. Previously, SIRT4 expression in colorectal cancer has been demonstrated to lead to a decrease in unfavorable clinical outcomes associated with this tumor $[10,11]$. However, the relationship between SIRT4 and prostate cancer remains to be elucidated. In this study, using immunohistochemical analysis, we revealed that the expression of SIRT4 in prostate cancer tissues was significantly lower than that in adjacent nonneoplastic prostate tissues. Besides, we found that patients with low expression levels of SIRT4 exhibited a higher Gleason score. In our in vitro study, by overexpressing and inhibiting SIRT 4 gene in prostate cancer cells, we demonstrated that SIRT4 could significantly inhibit the cell proliferation, migration, and invasion capabilities of prostate cancer cells. These findings suggest that SIRT4 exhibits significant tumor suppressing activities against prostate cancer cells. To the best of our knowledge, this study is the first study to reveal a significant association between SIRT4 protein expression levels and Gleason score in patients with prostate cancer.

Loss of apoptosis can impact tumor initiation, progression and metastasis[18]. Many studies have shown that the caspase family plays an important role in apoptotic cell death. Caspase-9, a member of the executioner family, which plays an important role in apoptotic incidents, whereas cleaved caspase-3 is a downstream protease of caspase-9 in the apoptosis pathway $[19,20]$. The nuclear factor-KB (NF-KB) family comprises five component subunits forming distinct transcriptionally active heterodimers or homodimers, including NF-KB1 and p65 (p105/p50), and displays anti-apoptotic effects [21], and the p65 subunit takes care of most of the roles of NF-KB [22]. In this study, we observed that SIRT4 overexpression increased the protein levels of caspase- 9 and cleaved caspase- 3 and inhibited NF-KB activity by reducing the nuclear translocation of p65. These results indicated that SIRT4 inhibits cellular proliferation of prostate cancer cells by inducing apoptosis.

One of the major characteristics of tumors is the varied energy metabolism [23]. Tumor cells have a distinct metabolic pattern compared with normal cells; they frequently appear to increase glutamine metabolism and glucose metabolism to facilitate cell growth $[24,25]$. Therapeutics obstructing the metabolic pathway of tumor cells is a novel tumor therapeutic method[26, 27]. For example, using glucose inhibitors to obstruct the glucose metabolism pathway has been considered as a useful tumor therapy method in studies[28]. However, tumor cells can also proliferate via the activation glutamine metabolism when glucose metabolism is inhibited for cellular survival. Thus, glutamine metabolism is important for tumor survival in glucose-lacking environment[29]. Besides, recent research discovered that 
lack of glutamine can induce KRas-driven S-phase arrest in cancer cells, which is caused by inadequate nucleotide biosynthesis [30]; besides, these arrested cells are more sensitive to cytotoxic drugs such as rapamycin, paclitaxel, and capecitabine [31-33], Consequently, blocking glutamine metabolism. Thus, inhibiting glutamine and glucose metabolism or administering synergistic chemotherapy drugs simultaneously, is a promising approach in cancer therapy [34]. Previous studies have found that SIRT4 can inhibit glutamine metabolism [35]. In this study, we showed that overexpression of SIRT4 downregulated the proteins associated with migration and invasion, and inhibited the proliferation and migration, and invasion capabilities of prostate cancer cells, and that these effects could be achieved by inhibiting the glutamine metabolism. These results indicated the therapeutic potential of SIRT4 for targeting glutamine metabolism in prostate cancer, especially in combination with glucose metabolism inhibitors.

To the best of our knowledge, this is the first study to demonstrate the tumor suppressive activity of SIRT4 in prostate cancer cells through inhibition of glutamine metabolism and induction of cell apoptosis in vitro. Notably, a significant association of SIRT4 expression levels with Gleason score was also revealed in patients with prostate cancer. Thus, SIRT4 may serve as a potential novel therapeutic target for prostate cancer.

\section{Declarations}

\section{Consent for publication}

Yes

\section{Availability of data and materials}

The datasets used and/or analyzed during the current study are available from the corresponding author upon reasonable request.

\section{Competing interests}

The authors declare that they have no known competing financial interests or personal relationships that could have appeared to influence the work reported in this paper.

\section{Funding}

This research is financially supported by the Zhejiang Natural Science Foundation (No. LY18H160055). The funding bodies played no role in the design of the study and collection, analysis, and interpretation of data and in writing the manuscript.

\section{Authors' contributions}

ALL the authors have read and approved the manuscript. 
LLC: Data curation; Formal analysis; Investigation; Writing - original draft

ZHG: Methodology, Visualization

YQX: Data analysis

PLS: Writing - review \& editing

GYH: Writing - review \& editing

\section{Acknowledgements}

None.

\section{References}

1. Aggarwal RR, Beer TM, Weinberg VK, Higano C, Taplin ME, Ryan CJ, Lin AM, Alumkal J, Graff JN, Nordquist LT, Herrera I and Small EJ. Intermittent Chemotherapy as a Platform for Testing Novel Agents in Patients With Metastatic Castration-Resistant Prostate Cancer: A Department of Defense Prostate Cancer Clinical Trials Consortium Randomized Phase II Trial of Intermittent Docetaxel With Prednisone With or Without Maintenance GM-CSF. Clin Genitourin Cancer 2015; 13: e191-198.

2. Siegel RL, Miller KD and Jemal A. Cancer Statistics, 2017. CA Cancer J Clin 2017; 67: 7-30.

3. Finkel T, Deng CX and Mostoslavsky R. Recent progress in the biology and physiology of sirtuins. Nature 2009; 460: 587-591.

4. Haigis MC, Mostoslavsky R, Haigis KM, Fahie K, Christodoulou DC, Murphy AJ, Valenzuela DM, Yancopoulos GD, Karow M, Blander G, Wolberger C, Prolla TA, Weindruch R, Alt FW and Guarente L. SIRT4 inhibits glutamate dehydrogenase and opposes the effects of calorie restriction in pancreatic beta cells. Cell 2006; 126: 941-954.

5. Nasrin N, Wu X, Fortier E, Feng Y, Bare OC, Chen S, Ren X, Wu Z, Streeper RS and Bordone L. SIRT4 regulates fatty acid oxidation and mitochondrial gene expression in liver and muscle cells. J Biol Chem 2010; 285: 31995-32002.

6. Ahuja N, Schwer B, Carobbio S, Waltregny D, North BJ, Castronovo V, Maechler P and Verdin E. Regulation of insulin secretion by SIRT4, a mitochondrial ADP-ribosyltransferase. J Biol Chem 2007; 282: 33583-33592.

7. Jeong SM, Xiao C, Finley LW, Lahusen T, Souza AL, Pierce K, Li YH, Wang X, Laurent G, German NJ, Xu X, Li C, Wang RH, Lee J, Csibi A, Cerione R, Blenis J, Clish CB, Kimmelman A, Deng CX and Haigis MC. SIRT 4 has tumor-suppressive activity and regulates the cellular metabolic response to DNA damage by inhibiting mitochondrial glutamine metabolism. Cancer Cell 2013; 23: 450-463.

8. Csibi A, Fendt SM, Li C, Poulogiannis G, Choo AY, Chapski DJ, Jeong SM, Dempsey JM, Parkhitko A, Morrison T, Henske EP, Haigis MC, Cantley LC, Stephanopoulos G, Yu J and Blenis J. The mTORC1 
pathway stimulates glutamine metabolism and cell proliferation by repressing SIRT4. Cell 2013; 153: 840-854.

9. Huang G, Cui F, Yu F, Lu H, Zhang M, Tang H and Peng Z. Sirtuin-4 (SIRT4) is downregulated and associated with some clinicopathological features in gastric adenocarcinoma. Biomedicine \& Pharmacotherapy 2015; 72: 135-139.

10. Miyo M, Yamamoto H, Konno M, Colvin H, Nishida N, Koseki J, Kawamoto K, Ogawa H, Hamabe A, Uemura M, Nishimura J, Hata T, Takemasa I, Mizushima T, Doki Y, Mori M and Ishii H. Tumoursuppressive function of SIRT4 in human colorectal cancer. Br J Cancer 2015; 113: 492-499.

11. Huang G, Cheng J, Yu F, Liu X, Yuan C, Liu C, Chen X and Peng Z. Clinical and therapeutic significance of sirtuin-4 expression in colorectal cancer. Oncol Rep 2016;

12. Jeong SM, Hwang S and Seong RH. SIRT4 regulates cancer cell survival and growth after stress. Biochemical and biophysical research communications 2016; 470: 251-256.

13. Lai X, Yu Z, Chen $X$ and Huang G. SIRT4 is upregulated in Chinese patients with esophageal cancer. Int J Clin Exp Pathol 2016; 9: 10543-10549.

14. Yeo W, Chan SL, Mo FK, Chu CM, Hui JW, Tong JH, Chan AW, Koh J, Hui EP, Loong H, Lee K, Li L, Ma $\mathrm{B}, \mathrm{To} \mathrm{KF}$ and Yu SC. Phase I/II study of temsirolimus for patients with unresectable Hepatocellular Carcinoma (HCC)- a correlative study to explore potential biomarkers for response. BMC Cancer 2015; 15: 395.

15. Azim HA, Jr., Peccatori FA, Brohee S, Branstetter D, Loi S, Viale G, Piccart M, Dougall WC, Pruneri G and Sotiriou C. RANK-ligand (RANKL) expression in young breast cancer patients and during pregnancy. Breast Cancer Res 2015; 17: 24.

16. Li W, Li S, Li Y, Lin X, Hu Y, Meng T, Wu B, He R and Feng D. Immunofluorescence Staining Protocols for Major Autophagy Proteins Including LC3, P62, and ULK1 in Mammalian Cells in Response to Normoxia and Hypoxia. Methods Mol. Biol. 2019; 1854: 175-185.

17. DeBerardinis RJ, Lum Jj Fau - Hatzivassiliou G, Hatzivassiliou G Fau - Thompson CB and Thompson $\mathrm{CB}$. The biology of cancer: metabolic reprogramming fuels cell growth and proliferation.

18. Lowe SW and Lin AW. Apoptosis in cancer. Carcinogenesis 2000; 21: 485-495.

19. Larsen BD and Sorensen CS. The caspase-activated DNase: apoptosis and beyond.

20. Creagh EM. Caspase crosstalk: integration of apoptotic and innate immune signalling pathways.

21. Ghosh S, May Mj Fau - Kopp EB and Kopp EB. NF-kappa B and Rel proteins: evolutionarily conserved mediators of immune responses.

22. Farhana L, Dawson Mi Fau - Fontana JA and Fontana JA. Apoptosis induction by a novel retinoidrelated molecule requires nuclear factor-kappaB activation.

23. Hanahan D and Weinberg RA. Hallmarks of cancer: the next generation. Cell 2011; 144: 646-674.

24. Daye D and Wellen KE. Metabolic reprogramming in cancer: unraveling the role of glutamine in tumorigenesis. Semin Cell Dev Biol 2012; 23: 362-369. 
25. Tennant DA, Duran RV and Gottlieb E. Targeting metabolic transformation for cancer therapy. Nat Rev Cancer 2010; 10: 267-277.

26. Pavlova NN and Thompson CB. The Emerging Hallmarks of Cancer Metabolism.

27. Weyandt JD, Thompson CB, Giaccia AJ and Rathmell WK. Metabolic Alterations in Cancer and Their Potential as Therapeutic Targets.

28. Abdel-Wahab AF, Mahmoud W and Al-Harizy RM. Targeting glucose metabolism to suppress cancer progression: prospective of anti-glycolytic cancer therapy.

29. Altman BJ, Stine ZE and Dang CV. From Krebs to clinic: glutamine metabolism to cancer therapy.

30. Gaglio D, Soldati C, Vanoni M, Alberghina L and Chiaradonna F. Glutamine deprivation induces abortive s-phase rescued by deoxyribonucleotides in k-ras transformed fibroblasts. PLoS One 2009; 4: e4715.

31. Mukhopadhyay S, Saqcena M and Foster DA. Synthetic lethality in KRas-driven cancer cells created by glutamine deprivation. Oncoscience 2015; 2: 807-808.

32. Saqcena M, Patel D, Menon D, Mukhopadhyay S and Foster DA. Apoptotic effects of high-dose rapamycin occur in S-phase of the cell cycle. Cell Cycle 2015; 14: 2285-2292.

33. Saqcena M, Mukhopadhyay S, Hosny C, Alhamed A, Chatterjee A and Foster DA. Blocking anaplerotic entry of glutamine into the TCA cycle sensitizes K-Ras mutant cancer cells to cytotoxic drugs. Oncogene 2015; 34: 2672-2680.

34. Le A, Lane AN, Hamaker M, Bose S, Gouw A, Barbi J, Tsukamoto T, Rojas CJ, Slusher BS and Zhang $\mathrm{H}$. Glucose-independent glutamine metabolism via TCA cycling for proliferation and survival in B cells. Cell metabolism 2012; 15: 110-121.

35. Mathias RA, Greco TM, Oberstein A, Budayeva HG, Chakrabarti R, Rowland EA, Kang Y, Shenk T and Cristea IM. Sirtuin 4 is a lipoamidase regulating pyruvate dehydrogenase complex activity. Cell 2014; 159: 1615-1625.

\section{Figures}



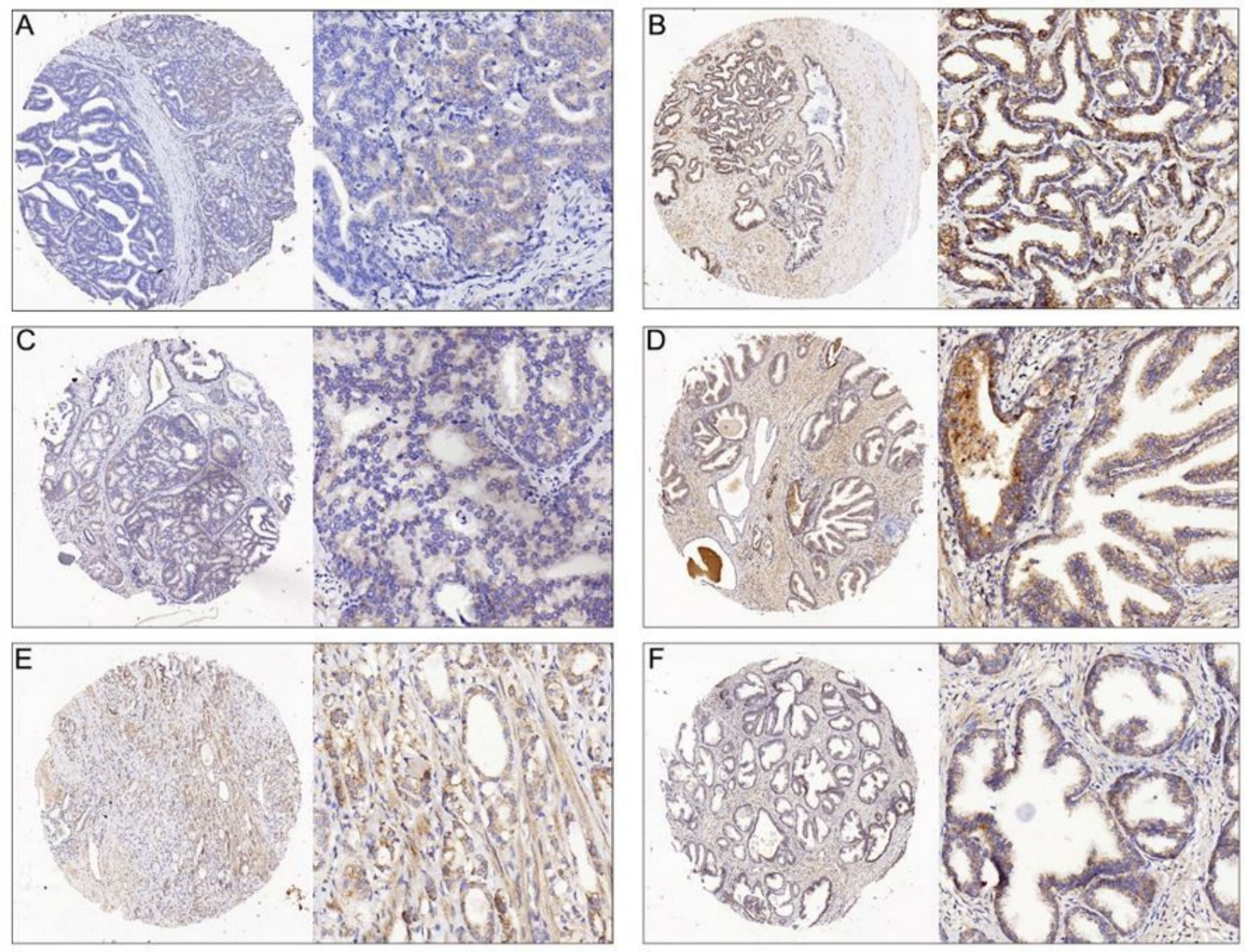

\section{Figure 1}

\section{Representative immunohistochemical staining of SIRT4 in prostate tumor cells}

SIRT4 is localized to the cytoplasm and is expressed at lower levels in tumor tissues as compared with adjacent non-neoplastic prostate tissues. The micrographs showed weak (A), median (C) and high (E) expression of SIRT4 in the prostate cancer tissues. The relevant expression of SIRT4 in corresponding adjacent non-neoplastic prostate tissuesin cases showing $A, C$ and $E$ were shown in $B, D$ and $F$, respectively (magnification: left panel 100x, right panel 400x). 
A

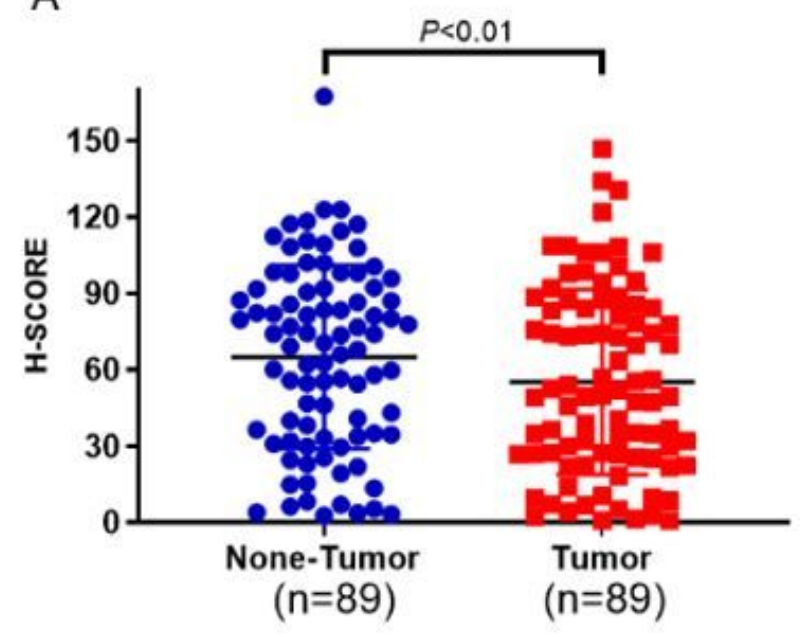

B

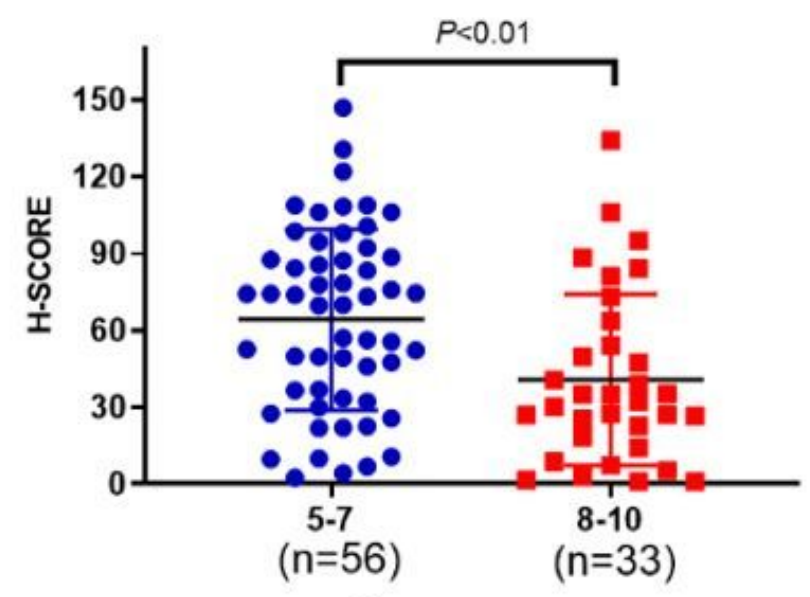

Gleason score

Figure 2

The statistical results of SIRT4 protein level differences and the association of immunohistochemical SIRT4 expression levels with Gleason score of patients with prostate cancer

(A) SIRT4 protein levels measured in 89 prostate cancer tissues and paired adjacent non-neoplastic prostate tissues using tissue microarray. SIRT4 protein levels are lower in tumor tissues compared with adjacent non-neoplastic prostate tissues $(P<0.01)$.

(B) SIRT4 expression is lower in prostate patients with higher Gleason score $(P<0.01)$.

The boxes represent the interquartile range; whiskers represent the 5th-95th percentile range, and bars represent the median. 
A

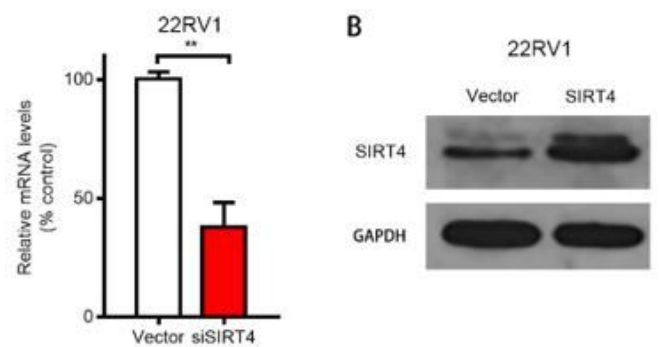

E

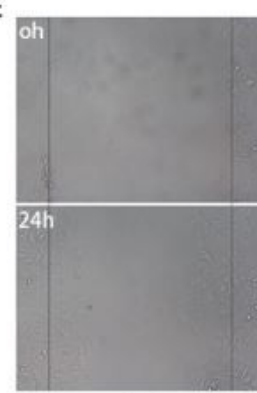

Vector

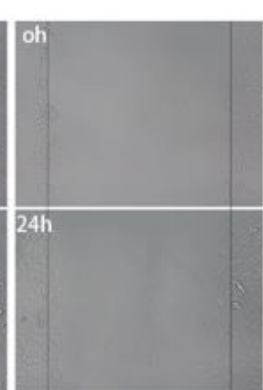

SIRT4

G
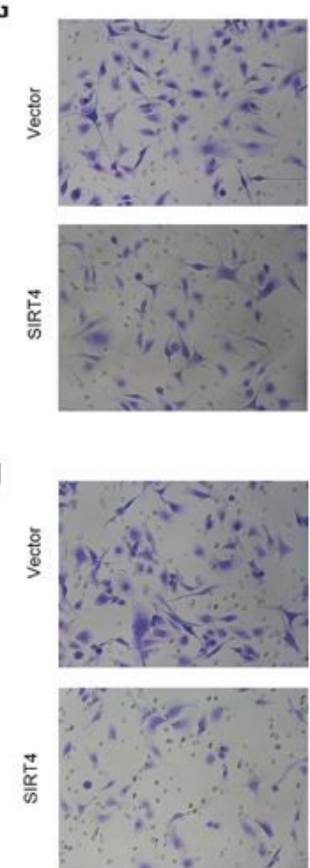
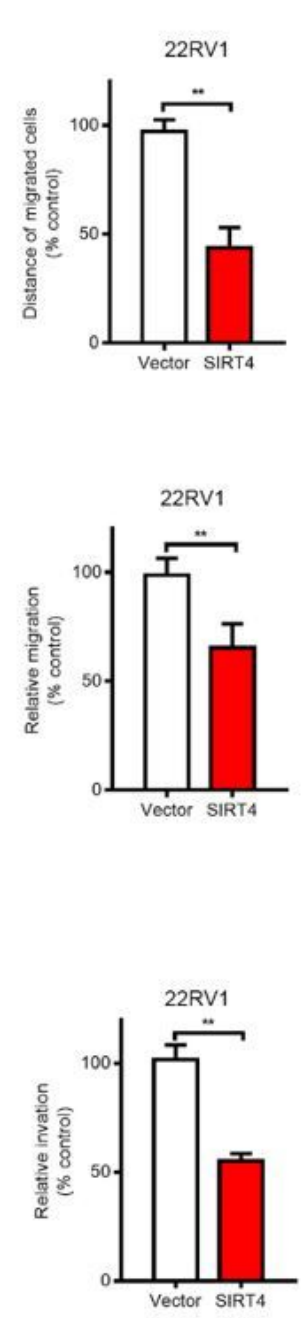

C

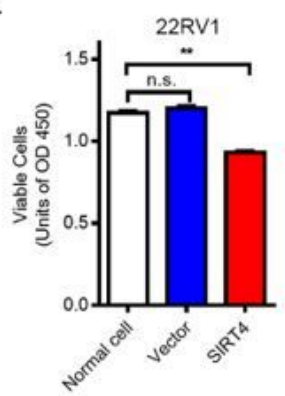

$\mathrm{F}$
D
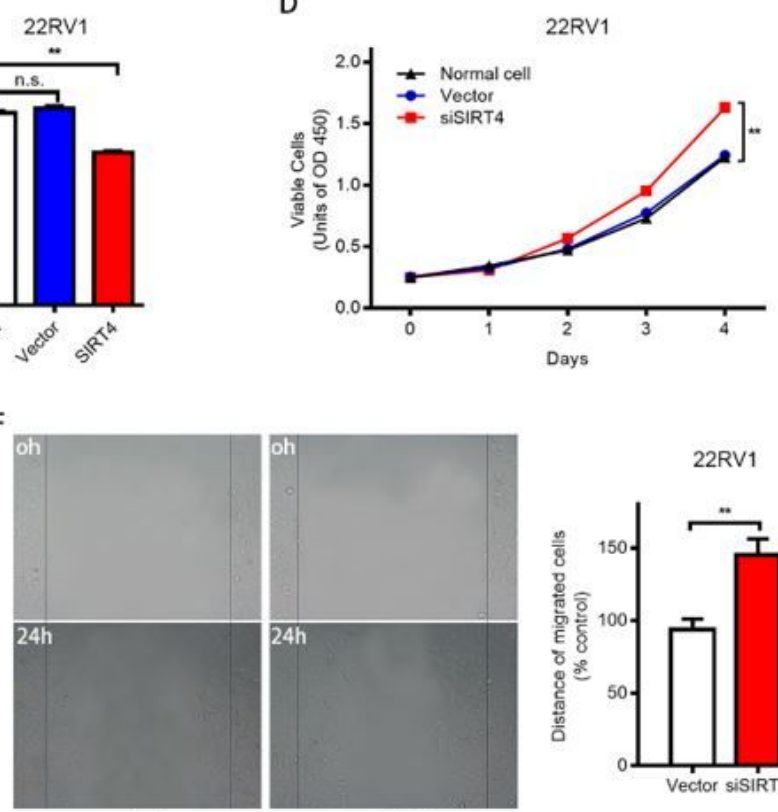

Vector

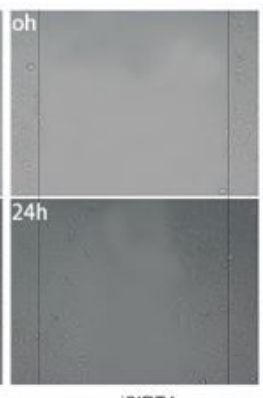

siSIRT4

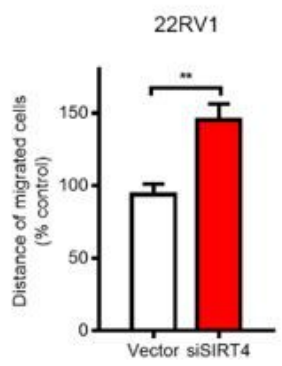

Vector sisIRT 4
H
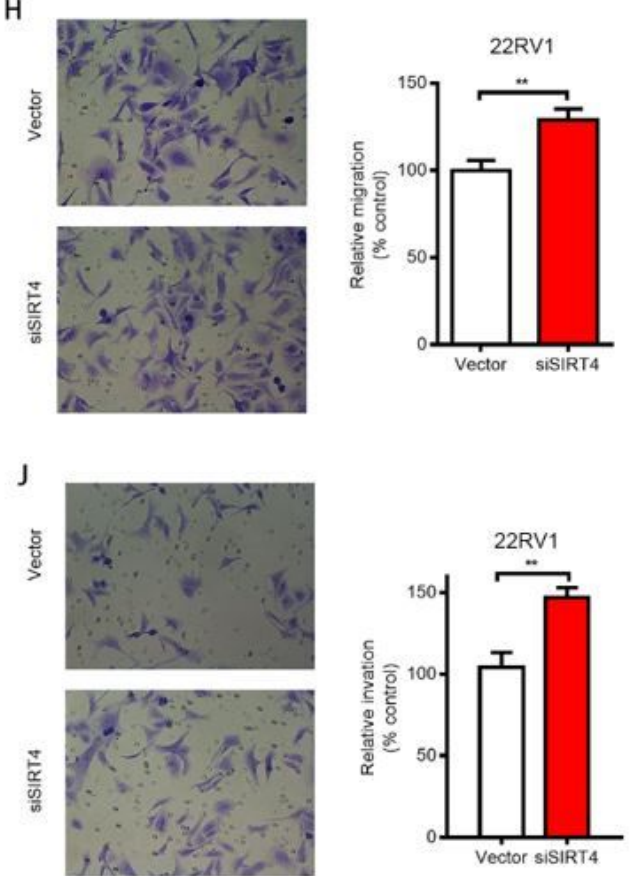

Figure 3

SIRT4 inhibits cell proliferation, migration and invasion capabilities of the prostate cancer cell

(A) 22RV1 cells transfected with vector or shSIRT4 are analyzed using qRT-PCR to evaluate SIRT4 expression.

(B)Western blot analysis of SIRT4 overexpression following treatment with puromycin $2 \mathrm{ug} / \mathrm{ml}$ for two weeks (right), GAPDH as an internal control. 
(C) The proliferation of vector and SIRT4-OE 22RV1 cells. Cell Proliferative activity is measured at $72 \mathrm{~h}$ after seeding.

(D) Proliferation curve of vector and shSIRT4 22RV1 cells. Cell Proliferative activity is measured every 24 $h$ for four consecutive days.

(E) Representative picture of Wound healing assay of vector and SIRT4-OE 22RV1 cells (left). The ratio of cell migration in SIRT4-OE cells to that of vector cells (right)

(F) Representative picture of wound healing assay of vector and shSIRT4 22RV1 cells (left). The ratio of cell migration in SIRT4-OE cells to that of vector cells (right)

( $G$ and I) The migration ( $\mathrm{G}$ ) and invasion (I) of 22RV1 cell as determined by transwell assays. Vector and SIRT4-OE 22RV1 cells are subjected to transwell assays. After being cultured for $18 \mathrm{~h}$ in transwell plates, the migrated or invaded cells are stained and counted under a microscope.

$(\mathrm{H}$ and $\mathrm{J}$ ) The migration $(\mathrm{H})$ and invasion $(\mathrm{J})$ of 22RV1 cell as determined by transwell assays. Vector and shSIRT4 22RV1 cells are subjected to transwell assays. After being cultured for $18 \mathrm{~h}$ in transwell plates, the migrated or invaded cells are stained and counted under a microscope. ${ }^{* * P<0.01}$ 
A
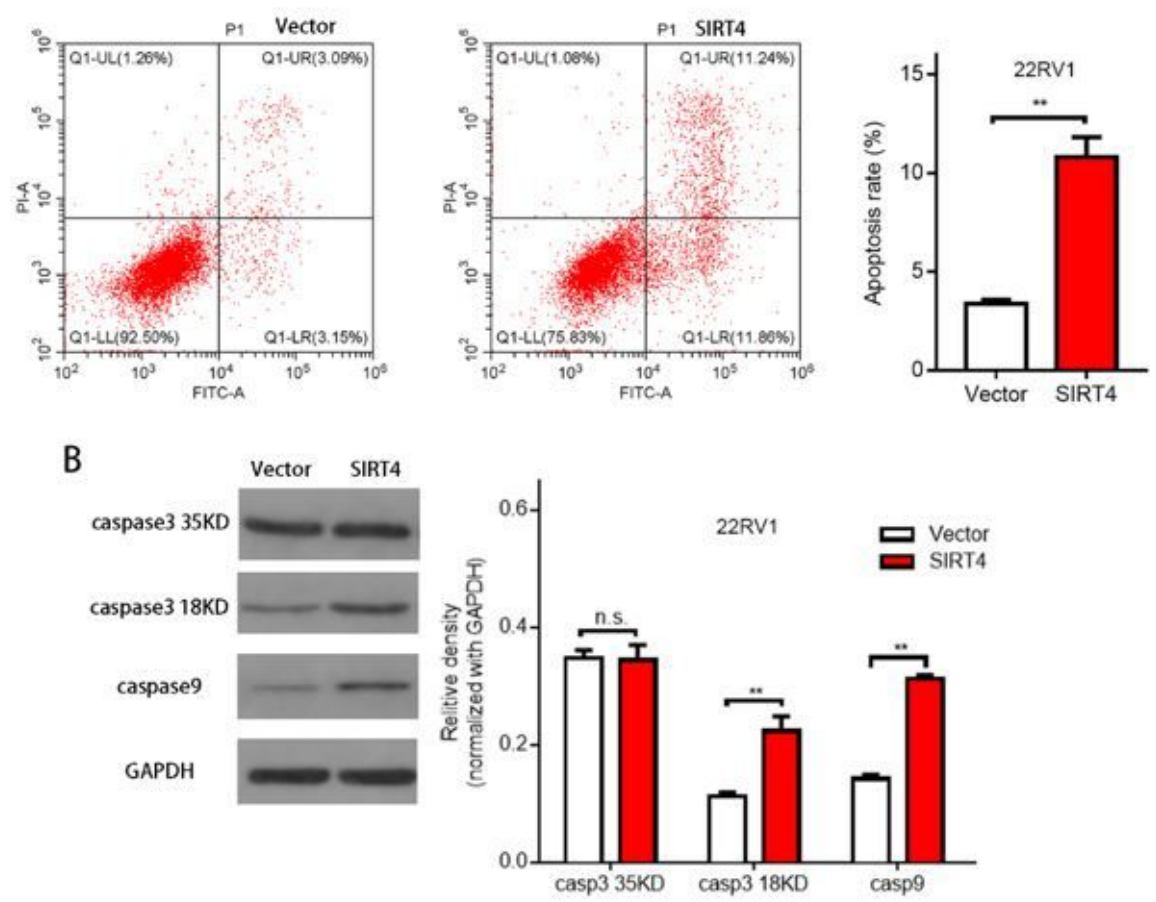

C

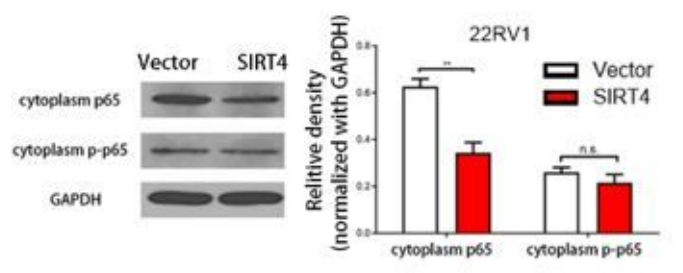

D

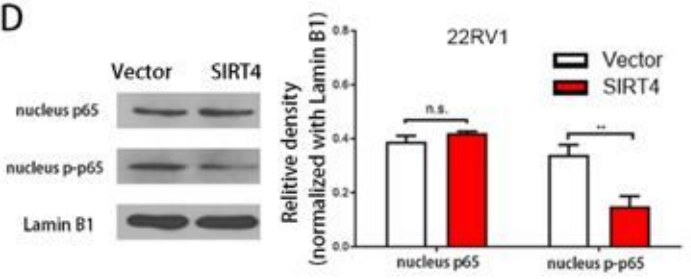

E

Vector
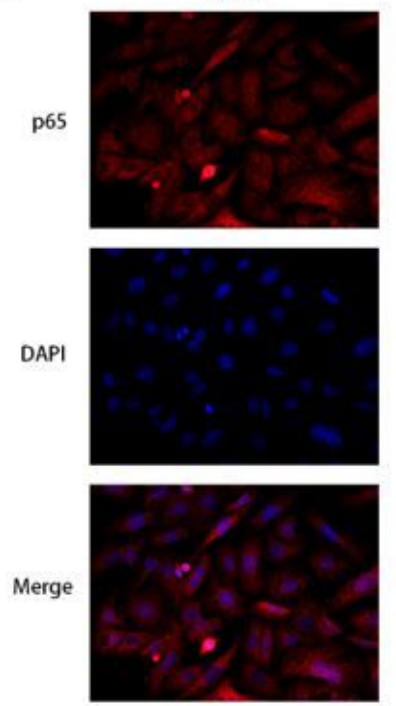

SIRT 4
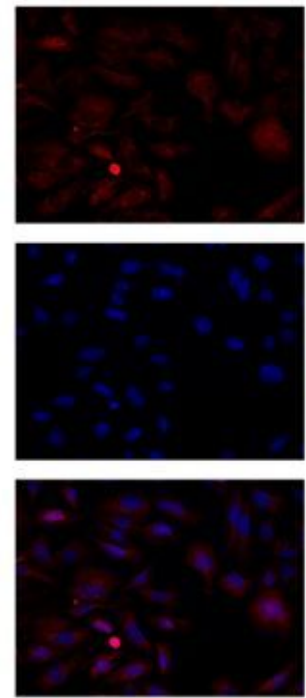

$\mathrm{F}$
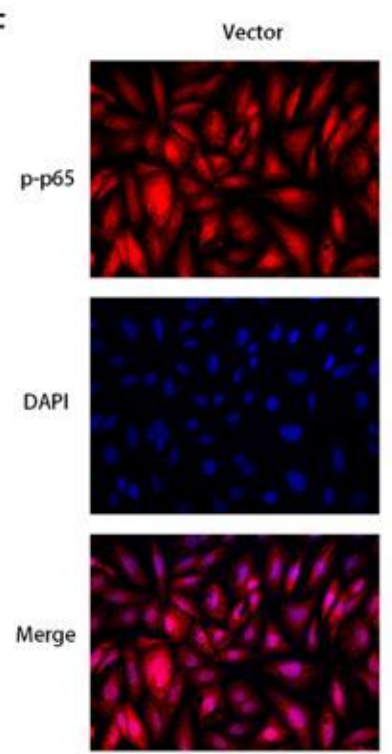

SIRT 4
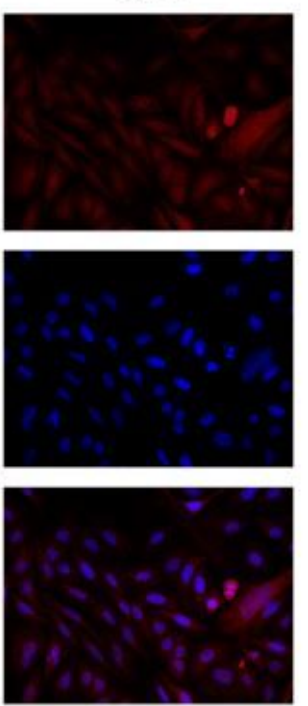

Figure 4

SIRT4 induces cell apoptosis through suppression of NF-KB activity by reducing the nuclear translocation of p65 in 22RV1 cells

(A) Vector and SIRT4 overexpression in 22RV1 cells is detected using FITC and PI staining with flow cytometry. 
(B) The relative density of caspase 3 and caspase 9proteins compared with GAPDH: density is analyzed and quantified using the Image $\mathrm{J}$ software. Results are representative of three independent experiments and are expressed as mean \pm SD.

(C) Effect of overexpression of SIRT4 on p65 and p-p65 protein levels in cytoplasm.

(D) Effect of overexpression of SIRT4 on p65 and p-p65 protein levels in the nucleus.

(E) The effect of overexpression of SIRT4 on p65 levels in 22RV1 cells as observed by immunofluorescence.

(F) The effect of overexpression of SIRT4 on p-p65 levels in 22RV1 cells as observed by immunofluorescence. ${ }^{\star} \mathrm{P}<0.05 .{ }^{\star *} \mathrm{P}<0.01$; NS, not significant. 

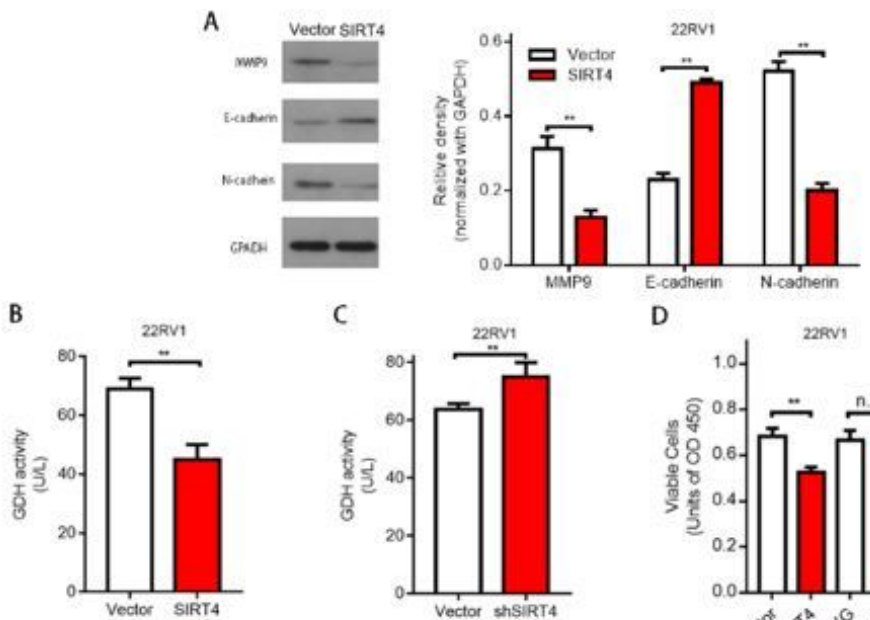

D 22Rvi

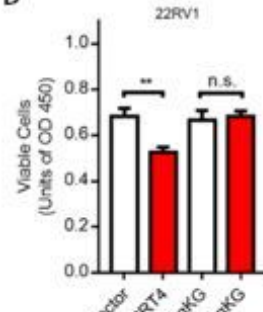

$\mathrm{E}$
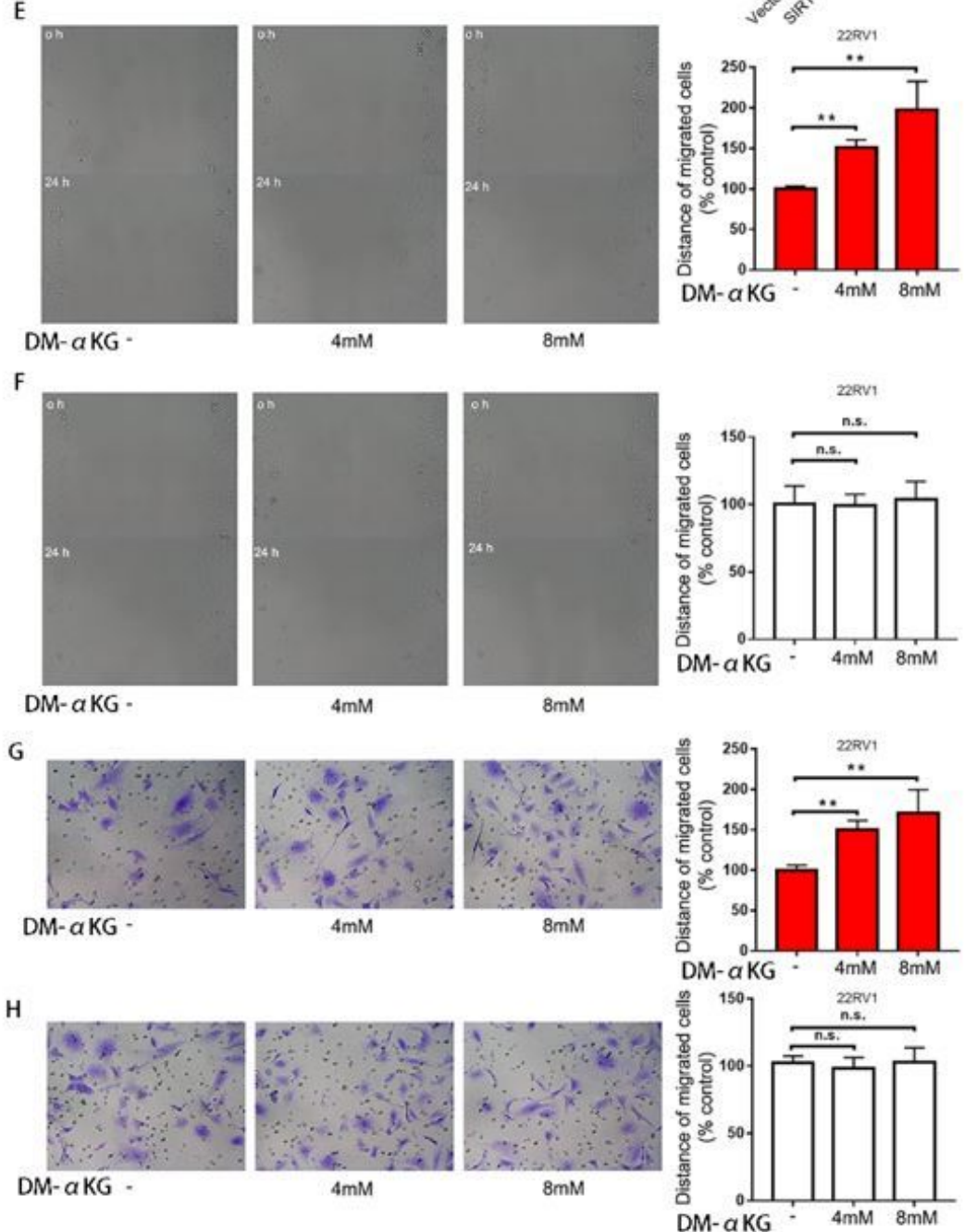

\section{Figure 5}

\section{Regulation of cellular mobility by SIRT4 via suppression of glutamine metabolism}

(A) The relative density of MMP9, E-cadherin, and N-cadherin proteins compared with GAPDH; density is analyzed and quantified using the Image $\mathrm{J}$ software. 
(B) Glutamate dehydrogenase activity in control and 22RV1 cells overexpressing SIRT4.

(C) Glutamate dehydrogenase activity in control and SIRT4 knockdown 22RV1 cells.

(D) The proliferation activity of control vector-transfected and SIRT4-overexpression in 22RV1 cells in stand media or media supplemented with DM-aKG $(8 \mathrm{mmol} / \mathrm{L})$. Cell proliferative activity is measured at $72 \mathrm{~h}$ after seeding the cell.

(E and F) The wound healing assay with SIRT4-overexpressing 22RV1 (E) and control cells (F) treated with DM-aKG for 0 and $24 \mathrm{~h}$, respectively. Representative images at the indicated time points are shown on the left (scale bar, $100 \mathrm{~mm}$ ).

( $G$ and $H$ ) The cell invasion assay in SIRT4-overexpressing 22RV1 cells (G) and control cells $(H)$ treated with DM-aKG after incubation for 0 and $24 \mathrm{~h}$, respectively. Representative images at the indicated times are on the left (scale bar, $100 \mathrm{~mm}$ ). Data are presented as mean \pm SD of at least three independent experiments. ${ }^{\star *} \mathrm{P}<0.01$; NS, not significant.

\section{Supplementary Files}

This is a list of supplementary files associated with this preprint. Click to download.

- SupplementarymaterialuncroppedWBimage.docx 